\title{
AMODS: Autonomous Mobile On-orbit Diagnostic System
}

\author{
Edward A.S. Hanlon, Benjamin P. Keegan, Benjamin H. Bailin, Jin S. Kang \\ United States Naval Academy \\ 590 Holloway Rd., MS 11B, Annapolis MD 21412; 410-293-6411 \\ kang@usna.edu
}

\begin{abstract}
Researchers at the United States Naval Academy are developing an Autonomous Mobile On-orbit Diagnostic System (AMODS) which utilizes CubeSats to perform on-orbit inspections on conventional spacecraft. AMODS comprises a multiple CubeSat arrangement wherein one self-propelled transport CubeSat (BRICSat) will deliver one of several "repair" CubeSats (RSats) with manipulable arms to spacecraft for the purpose of providing diagnostic, maintenance and repair services. AMODS will be validated in three phases. Phase One commenced in May 2015 with the launch of the transport module (BRICSat-P) where the effectiveness of microcathode thrusters to provide appropriate $\Delta \mathrm{V}$ has been evaluated. Phase Two, planned for 2017, involves a launch of the prototype repair unit, RSat, to test the effectiveness of the manipulable arms. Phase Three will combine the launch of the propulsion module, BRICSat and three temporarily linked repair modules, RSat-1,2,3. This paper provides an overview of the entire mission. It discusses the design of the BRICSat transfer vehicle, its required capabilities and the microcathode development results. It presents an analysis of RSat, its visual, assessment and repair proficiencies, the design strategy behind the manipulators' end-effectors and requirements for actuations in a space environment. Methods of validating and integrating systems on-orbit are also discussed.
\end{abstract}

\section{INTRODUCTION}

In 2004, the Estrela do Sul/Telstar 14 spacecraft suffered a crippling solar array failure. The cause was never established. ${ }^{1} \quad$ After spending $\$ 13$ million attempting to determine the root cause of the issue, Space Systems/Loral (SS/L) abandoned the effort. Seven years later, in 2011, the replacement spacecraft Estrela do Sul-2/Telstar-14R suffered a similar failure. SS/L spent an additional \$22 million troubleshooting the problem, ultimately tracing the flaw to a misplaced wire tie. ${ }^{1}$ Yet in 2012, the solar panels on SS/L built IS19 also failed. SS/L reopened their study of the root cause, finally concluding that defects in the honeycomb structure of the solar panel caused explosive decompression, destroying the panels. ${ }^{1}$

Had SS/L been able to capture images of the failed 2004 spacecraft, their diagnostic analysis could have been much less expensive, and effective remedial revisions could have been made to the two subsequent missions to prevent similar malfunctions. SS/L President John Celli provided this justification for the delay in establishing the cause: "You can only act on the data you have. In 2004 we did not have the same data as we did in 2012. We have never before seen a ground failure of our solar arrays." ${ }^{\text {Real time images }}$ could have provided SS/L with the data they needed to realize that the spacecraft's flaw was not with the deployment mechanism, as originally assumed, but with the panel itself. Unfortunately, after a spacecraft is launched, it is nearly impossible to physically interact with it again.

Extensive work has been done to attempt to bridge this capability gap. Some missions are optimized for human servicing, most notably the Hubble Space Telescope (HST). HST benefited from five shuttle maintenance and upgrade missions, with great success. While suitable for a flagship space telescope of almost incalculable scientific value, these missions put human lives at risk and remain extremely expensive. ${ }^{2}$

Attempts have been made to remove the human element; DARPA's Orbital Express, sought to validate a spacecraft's ability to refuel and reconfigure on-orbit satellites. The U.S. Air Force launched two demonstration spacecraft in 2007, and validated much of the concept.

The Satellite Servicing Capabilities Office (SSCO, NASA Goddard) has provided both a conceptual robotic repair mission and some of the components needed to undertake it. These components are currently undergoing testing on the International Space Station as part of the Robotic Refueling Mission, and are operated by the Dextre robot. ${ }^{3}$

The common denominator across all of these missions is a high cost-Shuttle repair missions were valued at $\$ 900$ million, while Dextre cost $\$ 200$ million and Orbital Express cost $\$ 300$ million. ${ }^{2,3,4}$ They all provide 
almost assured repair or refueling and for some marquee missions this capability is worth the price.

However, SS/L did not need to repair Telstar 14-even knowing about the nature of the failure would have saved nearly $\$ 300$ million. ${ }^{1}$ The U.S. Naval Academy's (USNA) focus has been on providing this relatively simple diagnostic capability, and not the "kitchen sink approach" favored by larger, more expensive spacecraft.

The goal of the Autonomous Mobile On-orbit Diagnostic System (AMODS) project is to assure the ability to provide the physical on-orbit interaction needed to generate diagnostic data. AMODS employs a modular, CubeSat style design approach to overcome traditional cost and technological hurdles.

This paper will provide an overview of the entire program, beginning with a discussion of the concept of operations, and continuing with a discussion of the multistep development process and design decisions that make the mission practical.

\section{MISSION SUMMARY}

The concept embraces a multiple CubeSat system: 1) several "repair" CubeSats (RSats) with manipulable arms designed to latch onto a host satellite and maneuver around, imaging and potentially repairing various components; and 2) one self-propelled transport CubeSat (BRICSat), a "space tug" with the ability to manage $\Delta \mathrm{V}$ and rendezvous operations.

\section{RSat Summary}

RSat is a $3 \mathrm{U}(10 \times 10 \times 33 \mathrm{~cm})$ cube satellite with two $60 \mathrm{~cm}$, seven degree of freedom robotic arms. It is intended to operate in constant contact with a host spacecraft. The robotic arms provide access to any external surface of the host. RSat will be equipped with a suite of equipment, including a camera to diagnose any on-orbit failures and, in some cases, perform minor on-orbit repairs or maintenance. RSat provides ground controllers with the continued opportunity to physically interact with their spacecraft as if it was on the ground. The projected cost is less than $\$ 25,000$.

\section{BRICSat Summary}

BRICSat is also a $3 \mathrm{U}$ CubeSat. Designed with its own propulsion system, it is intended to rendezvous with other spacecraft. It is a complement to RSat, and provides the only propulsive force to the RSat platform. BRICSat defrays the cost of expensive attitude control, rendezvous and propulsion systems across multiple RSats, greatly reducing the cost of deploying RSat across an existing constellation.

\section{CONCEPT OF OPERATIONS}

Conceptually, one BRICSat will be launched with multiple RSats (RSat-1, 2, 3, etc). These RSat will be free-floating in space in relatively close proximity, forming a loose "depot" of RSats. Once on-orbit, RSat1 will grapple itself to BRICSat which will transfer the RSat to its host satellite. At that point, RSat-1 will latch on to the client spacecraft and use its manipulators to propel itself around the craft providing visual data and other diagnostic function. Thereinafter, BRICSat will return to the depot, and transfer RSat-2 and later RSat-3 to their respective spacecraft hosts. The RSats will remain on their hosts, monitoring the satellites, visually documenting any features of interest and performing diagnostic and repair tasks as needed.

There are two types of missions the AMODS program will support: RSat deployment on future spacecraft and RSat deployment to existing on-orbit spacecraft.

\section{New (Future) Spacecraft Incorporating RSat}

RSat can be incorporated directly into the design of new spacecraft. This will allow RSat to launch alongside, or within, its host, rather than require delivery by BRICSat. A few modifications to potential host satellites will be needed to simplify RSat operations.

1. Modified P-POD: The P-POD container is the vessel from which CubeSats are traditionally deployed, and can be conceptualized as a box with a push-spring. Typically, a CubeSat is ejected into space from the P-POD container when the door opens. Rather than simply being pushed out into space, RSat will be temporarily fixed to the spring, allowing RSat to slide out on a drawer.

2. Contact Points: Where possible "grab" points will be situated around the host spacecraft to ease robotic maneuvering. Ideally, these locations will be located $1 \mathrm{~m}$ apart. They will consist of a small flange of metal, with a small lip, which will allow for confident manipulator capture.

3. Known Design: RSat will be pre-loaded with a structural and locational knowledge of the host satellite, translated from a CAD model of the spacecraft upon which it is operating. This will allow RSat to intelligently plan out its maneuvers and facilitate autonomous navigation operations.

On launch, RSat will be embedded in the spacecraft in the modified P-POD container. Immediately after the spacecraft reaches orbit, or when the host satellite 
desires, RSat will be deployed. Figure 1 shows a depiction of this deployment concept.

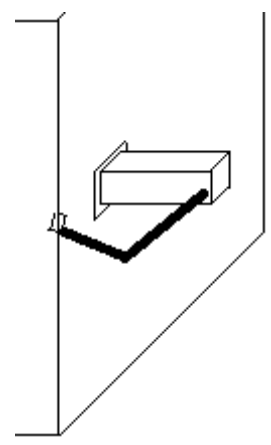

Figure 1: RSat Deployment

After the drawer upon which RSat sits slides out of the P-POD, RSat will commence a series of system diagnostics. At the conclusion of these tests, RSat will grab onto a contact point on the host spacecraft, and await commands from the ground. It will broadcast a periodic message that details its status.

At this point, it will enter a standby mode and wait for components of its host to fail. In the event a failure occurs, RSat will be awoken by ground controllers and commanded to navigate to the point of failure using its robotic arms to "spider" its way along.

RSat may also be used for periodic inspections to monitor system health and anticipate or even mitigate failures. It can provide detailed imagery of the spacecraft at a certain interval to detect events such as micrometeorite impacts, sputtering, and radiation effects. This could provide ground controllers a better sense of the state of health of their spacecraft.

After reaching the targeted failure location, RSat will perform a detailed analysis of the situation, and if possible, attempt to make repairs. Otherwise, it will downlink data on the failure so that ground controllers can learn exactly what has failed and how in order to prevent future failures.

\section{Servicing Existing Spacecraft}

The existing spacecraft mission assumes an AMODS will be deployed on a large constellation of satellites in similar orbits. A fleet of RSats will be launched: for every four to nine RSats there will be one BRICSat. This assembly of CubeSats would launch as a standard CubeSat Launch, possibly even as a secondary payload. After achieving orbit, BRICSat would navigate to a point near the closest RSat. RSat would then use its robotic arm to link with BRICSat. This is represented in Figure 2.

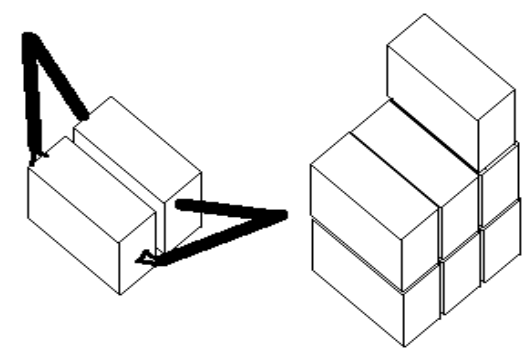

Figure 2: BRICSat-RSat Docking

BRICSat would then navigate the combined BRICSatRSat system to the client spacecraft. The unoccupied arm of one of the RSat will make contact with the client spacecraft. After confirmed capture, this first RSat will disconnect from BRICSat and conduct maneuvers as described in the "New Spacecraft" section above.

BRICSat will return to the depot and capture the next RSat for delivery to the next payload.

\section{BRICSat DESIGN}

The mission of the BRICSat platform is to provide the services needed to deploy RSat onto a distributed network of spacecraft. In order to rendezvous with the client spacecraft, it must be able to provide:

1. Propulsion: Provide a combination of long term, sustained $\Delta \mathrm{V}$ for travel between spacecraft, and quick pulses to allow for proximity operations.

2. Attitude Control: BRICSat must be able to provide ADCS functionality to RSat, as RSat will not need that capability when it is attached to its host. BRICSat must also be able to mirror any movements of the host spacecraft.

3. Navigation: BRICSat will need both a long range navigation system (GPS), and a short range system to handle the final approach to the target spacecraft.

BRICSat must also function as a completely independent spacecraft. In order to validate that BRICSat can provide this functionality, it will undergo a two stage test program. (P: Prototype and D: Demonstrator)

\section{BRICSat-P Test Vehicle}

The BRICSat-P spacecraft is a technology demonstrator designed to validate a portion of the AMODS propulsion system. BRICSat-P's propulsion system was designed by George Washington University. The first iteration of the satellite was launched in May 2015. 
A depiction of the propulsion system's thruster head is shown in Figure 3.

BRICSat-P demonstrates the "in transit" propulsion system and Attitude Control System portions of the overall BRICSat system. It is a $1.5 \mathrm{U}(10 \times 10 \times 16 \mathrm{~cm})$ spacecraft with four Micro-Cathode Arc Thrusters ( $\mu$ cat).

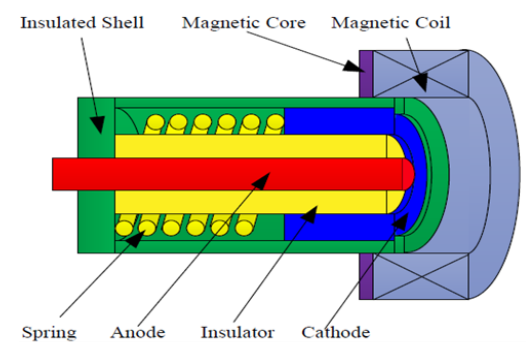

Figure 3: GWU Thruster Head

The thrusters are a form of electronic propulsion which utilizes a titanium cathode propellant to provide a specific impulse of $3000 \mathrm{~s}$ with a thrust of $1 \mu \mathrm{N} .^{6}$ An electronic arc creates a cathode spot that ablates the cathode to produce high velocity plasma. Thrust level is controlled by increasing or decreasing the firing rate.

When firing continuously, the thrusters draw one watt and can last for 10 years, depending on the size of the propellant rod. ${ }^{7}$ The thrusters are compact; $1 \mathrm{~cm}$ in diameter and $2.3 \mathrm{~cm}$ long. This allows them to be placed in optimal locations on the spacecraft, and allows the full size BRICSat to contain significantly more thrusters.

The BRICSat-P mission is the first flight test of these thrusters. The mission will conduct three primary flight experiments:

1. Detumble: BRICSat will use its thrusters to detumble, demonstrating the thrusters ability to act as an attitude control system.

2. Rotation: The rotational experiment uses the thrusters to rotate the spacecraft up to $6 \mathrm{rpm}$, which will evaluate the thrusters performance against known quantities.

3. Delta V: BRICSat-P will use its thrusters to change its orbit.

The results of these experiments will affect further development of the BRICSat product line.

\section{BRICSat-D Technology Demonstrator}

BRICSat-D is the evolution of the BRICSat genus. It will incorporate both the long term thrusters tested on the BRICSat-P flight and the maneuvering thruster system, in order to demonstrate rendezvous operations.

Based on the results from the $\mathrm{P}$ flight, flight D's propulsion system will consist of 12 micro-cathode thrusters, used for long duration cruise and coarse attitude control/pointing, coupled with a single cold gas thruster.

In operation, the $\mu$ cat thrusters and reaction wheels will orient the spacecraft alongside the first RSat. After both spacecraft are linked, the cold gas thruster will be briefly fired to create safe separation from the other RSats. The $\mu$ cat thrusters will then be used to navigate to the client spacecraft, where the cold gas thrusters will be used to handle any terminal guidance.

For the demonstration flight, BRICSat-D will chase another CubeSat (RSat-P, depending on launch arrangements), and demonstrate transit and rendezvous operations.

If the BRICSat-D flight proves successful, this design will be used as the master for all subsequent BRICSat production vehicles.

\section{RSat DESIGN}

The mission of the RSat spacecraft is to provide a mobile platform to survey and possibly repair a much larger spacecraft. It must be able to:

1. Locomote: RSat must be able to interact with every area of the spacecraft.

2. Investigate: The data returned must be useful to facilitate maintenance and failure analysis.

In addition, it must meet the following qualifications:

1. Price: In order to be competitive, an entry level RSat shall cost no more than $\$ 25,000$.

2. Accurate: RSat manipulators must be accurate enough to "do no harm."

In order to validate RSat's ability to provide these functions, RSat will undergo a two stage test program. (P: Prototype and D: Demonstrator)

\section{RSat-P Test Vehicle}

The first launch (targeted for early 2017) will be a prototype. It will fly a mission similar to a typical CubeSat and will float freely in space. The mission is 
designed as a project demonstration. On-orbit, it will prove RSat's suitability, capability, and accuracy. RSat will conduct on-orbit performance assessments by moving its appendages through a test pattern or patterns intended to simulate simple diagnostic or repair tasks.

\section{Spacecraft Systems}

RSat-P will be launched into low earth orbit, making the low-cost bus a viable option. The low-cost bus consists of a homegrown power supply system, two COTS amateur data radios, three COTS TTL serial cameras, and four Arduinos.

\section{Resilience}

Despite the low-cost COTS hardware, it is designed as an extremely robust system. This high resilience is made possible because RSat subdivided into two systems. Each Arm (Arm1 and Arm2) has its own microcontroller pair, radio, camera, and electrical power system (EPS). Some of these subsystems are capable of cross linking to the other arm as well to provide redundancy.

\section{Command and Data Handling}

All four main processors listen to both communication rails (that is both radios), but only execute commands when their specific function is tasked. They also have crosslinking capability via I2C to assume most of another processors' functionality.

\section{Communications}

RSat has two onboard radios, operating at the same frequency. Under typical operations, one operates as an intermittent beacon, while the other is used for high bandwidth tasks. After a set period of time, these radios rotate those duties, allowing for communication even if one has failed.

\section{Electrical Power System (EPS)}

A bespoke EPS allows for a near fully redundant EPS design. EPS control and telemetry can be provided from any functioning processor, while each arm has its own voltage converters and fuses-however all components are sized 2x max current draw, allowing a single component to drive both arms. No single component exists in the EPS that could cause complete spacecraft failure.

\section{Attitude Determination and Control System (ADCS)}

While the final variant of RSat will not require an ADCS system, such a system will be needed for the initial free floating launch. In order to operate the arms, RSat must detumble to a rotation rate of less than $1 \%$ s. To facilitate this, RSat will use a passive magnetic hysteresis system that favors the hysteresis material over a permanent magnet.

\section{$\underline{\text { Robotic Systems }}$}

The robotics subsystem is the primary facet of interest on RSat. The robotics system must be accurate to within one $\mathrm{cm}$ at full extension. This equates to $\pm 0.25^{\circ}$ at each motor. Actuations must be smooth and controlled to avoid damaging the host spacecraft. Materials and lubricants chosen must be space rated or at least space suited.

\section{Motor Selection}

The core of the robotics system is the motor selection. A vast quantity of motors was examined, and samples of several types were lab tested for accuracy. More information of the results can be obtained by contacting the corresponding author.

After minimizing motor diameter and motor length for the given accuracy, and specifying the need for zero "backlash," only one motor met the AMODS requirements: A $10 \mathrm{~mm}$ diameter, $40 \mathrm{~mm}$ long stepper/encoder/gearhead combination. This motor is available with a vacuum safe lubricant, which simplifies the assembly process.

\section{Robotics Porch}

In order to maximize the length of the arms, some deviations from the "traditional" CubeSat frame were required. A "front porch" design was adapted, which removes one face of the Cube, while maintaining the rails, to allow the arms to rotate outward. This " $A$ " frame design is shown in Figure 4. RSat's Robotics Bay is also shown in Figure 5.

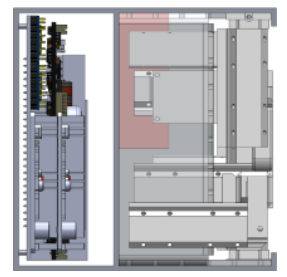

\section{Figure 4: RSat Cross Section}

\section{Arm Layout}

The "porch" allows the each arm to utilize the full length of the spacecraft, maximizing the overall length of each arm. A bi-fold design was selected to allow a maximum arm span of $1.5 \mathrm{~m}$. 
To ease construction and simplify the overall design, the motor unit directly drives the arms. This is primarily possible due to the gearhead built into the motors. It also allows for near complete modularity.

There is space left behind the arm segments that is not occupied by the motors. This is used for some power and communications equipment.

\section{Arm Material}

In order to maximize space and design efficiency, the arms are developed and constructed using rapid prototyping technology. This allows for additional fast printing of parts for vibration and vacuum testing, as well as for intricate shapes to mitigate some of these effects.

\section{Prototype Mission}

All of these components will be tested on-orbit to validate the accuracy and reliability of the arm system. There are four primary flight demonstrations:

1. Navigate to Coordinate: The purpose of the coordinate section is to demonstrate that each of the arms is capable of navigating to a precise location, which will indicate that the spacecraft is capable of flexible orbital operations. This is shown in Figure 5.

2. Handshake: The "Handshake" maneuver will demonstrate that RSat is capable of operating the arms in proximity to each other, as shown in Figure 6. This is a key requirement in any potential imaging/servicing missions.

3. Imaging: The "Imaging" portion of the demonstration phase establishes RSat's ability to take pictures of other spacecraft. RSat's arms will move to a variety of positions around the spacecraft and image all six faces. Figure 7 shows RSat imaging its $+Y$ face.

4. Manipulation: This mode simulates the use of the manipulators to interact with another spacecraft. Arm 1 will pick up a demonstration object from one of the ends of the spacecraft, and move it to within camera range. Arm 2 will then take control of the object. This validates the manipulator design, and demonstrates the precision of the arm. Figure 8 shows the manipulation movements of RSat arms.

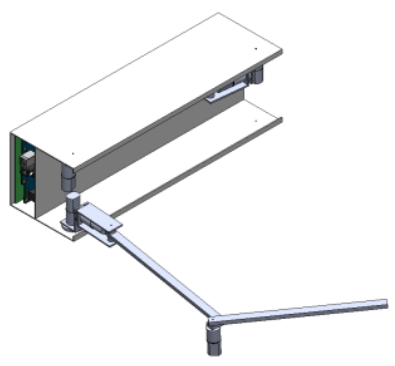

Figure 5: Navigate to Coordinate

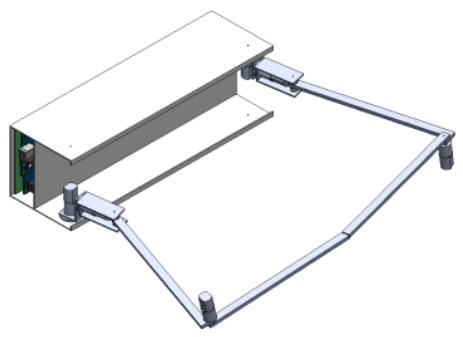

Figure 6: Handshake

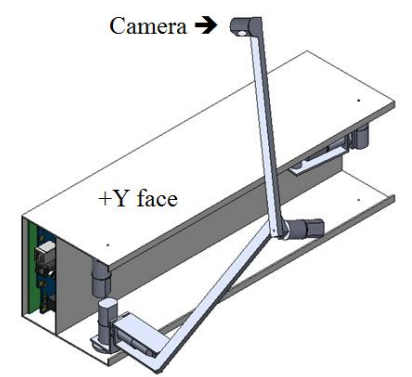

Figure 7: Imaging the $+Y$ Face
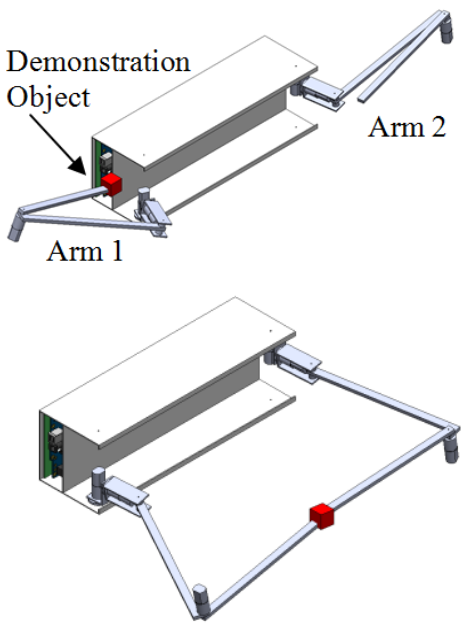

Figure 8: Manipulation 


\section{RSat-D Technology Demonstrator}

The current plan for the subsequent development is for the RSat-D technology demonstrator to be deployed on a second stage of a rocket after primary payload separation and will move around the stage as it would on a host satellite. This second flight test will demonstrate proximity operations, further establishing flight heritage and reliability.

This technology demonstrator will include refinements from the $\mathrm{P}$ design; it will utilize previously unused payload space for tool stowage to provide increased arm functionality, and will demonstrate the advanced radiation hardened processors to conduct some maneuvers autonomously.

\section{CONCLUSION}

Providing a spacecraft with on-orbit assessment and repair capability will increase the success rate of missions by both facilitating improved correlation between design and reality and providing immediate failure analysis and mitigation activates. The AMODS project is intended to provide this function on a cost efficient basis. After both BRICSat-D and RSat-D have flown, the AMODS concept will be validated. At this point, AMODS can commence deployment onto satellites, both new and legacy. Currently, the RSat-P project is midway through the development process and tracking to a 2017 launch, while BRICSat-P is being demonstrated in space at time of writing. Fundamentally, the system has the potential to demonstrate an inexpensive, reliable repair system that can be placed on any spacecraft. This technology will prove invaluable to extending the life of spacecraft and transitively the effectiveness of humans in space.

\section{ACKNOWLEDGEMENTS}

The authors gratefully acknowledge the generosity of Penny and Roe Stamps and the Stamps Family Charitable Foundation.

The team would also like to acknowledge with appreciation the contributions of Mr. Robert Ruark, CDR Robert Bruninga, USN (ret), Dr. Bradley Bishop, and CAPT Shorr, USAF.

\section{REFERENCES}

1. De Selding, P.B. "Spate of Solar-array Failures on SS/L Satellites Traced to Manufacturing Defect," SpaceNews, Alexandria, VA, 4 January 2013

2. Berger, B. and C. Parks, "NASA's Mission to Service Hubble in 2008 Will Cost $\$ 900$ Million” 31 October 2006.

3. NASA, "Robotic Refueling Mission," NASAfacts, Greenbelt, MD.

4. Berger, B. "U.S. Air Force to End Orbital Express Mission," Space.com, Washington, D.C. 30 June 2007.

5. Leary, W.E. "A Space Robot With Arms to Make R2D2 Jealous," New York Times, New York, New York, 11 March 2008.

6. Keidar, M. "Micro-Cathode Arc Thruster for PhoneSat Propulsion," 27th Annual AIAA/USU Conference on Small Satellites, Logan, UT, August 2013.

7. Dinelli, K "BRICSat-P "Ballistically Reinforced Communications Satellite," AIAA/SAE/ASEE Joint Propulsion Conference, July 2014. 